\title{
Experimental study on dynamic deformation of free surface under surrounding gas flow
}

\author{
Ruquan Liang ${ }^{1}$, Xiaoyuan $\mathrm{Li}^{1}$, linyang Zhu ${ }^{1}$, Zhihui Zhang ${ }^{1}$ \\ ${ }^{1}$ Key Laboratory of National Education Ministry for Electromagnetic Processes of Materials, Northeastern University in Shen Yang, CN
}

\section{Introduction}

The dynamic deformation of the free surface of liquid bridge is closely related with production of high-quality single crystal in floating zone method. Therefore, the conclusion driven by this paper can directly apply to the growth of melt crystal. In physical experiments, we use the ideal isothermal model to improve the quality of silicon crystal. Deformation of an isothermal interface is extremely related to a co-axial gas flow when enter from the bottom to the top, which was discussed by Gaponenko $^{[1,2]}$, and they reported that the linear velocity of gas almost determined the free surface deformation. The tendency that surface deformation grows with the volume ratio $V$ quasi-linearly was predicted by Gaponenko ${ }^{[3]}$. After a slow decaying of the period, the new oscillations are generated. The new liquid bridge decayed much faster. A linear dependence is not observed for all volume ratios, despite the small Reynolds number $\left(280<R e_{g}<560\right)$ is studied by Matsunaga ${ }^{[4]}$. The dynamic deformation displays a strong dependence on the liquid volume ratio and the direction of the gas stream parallel to the interface. The shape of liquid bridge which exists between two fixed support columns is usually maintained to be cylindrical by surface tension. In this paper, the experiment is carried out about dynamic deformation of free surface under the normal gravity environment. The object of this experiment is to study the adiabatic gas-liquid two-phase flow system with isothermal and cylindrical geometry. The dynamic deformation of the free surface of the liquid bridge is studied.

\section{Experiment Establishment and Experiment Process}

The equipment includes a high-speed camera, a liquid bridge support frame, a astigmatism film and a back light. The liquid bridge generation equipment is fixed on the horizontal experimental platform to adjust the liquid bridge to proper height. The micro-syringe is used to control the primary volume of silicone oil. The cylinder the sleeve, the white back light, the camera and the liquid bridge generation equipment are all set to be ready. Then, the gas enters the cylinder sleeve, after the ventilation is stable; the high-speed camera begins to shoot the shape of liquid bridge. The coordinate point data of liquid bridge surface is obtained by using boundary extraction technology and numerically simulated by ORIGIN software to form the figures, and the dynamic deformation of free surface of liquid bridge is acquired. In the experiment, the aspect ratio of liquid bridge is defined as $\Gamma=d / R_{0}$, where $\mathrm{d}$ and $R_{0}$ denote the height and support radius, respectively. Figure 1 is a simple diagram of the experiment.

The liquid bridge is surrounded by a vertical transparent glass sleeve with $R_{0}=3 \mathrm{~mm}$ and $R_{\text {out }}=5 \mathrm{~mm}$ remaining unchanged. Gas enters the glass sleeve from the lower part and flows through the annular tube of size.

$$
\begin{gathered}
R_{h}=2 \quad\left(R_{\text {out }}-R_{0}\right) \\
R e_{g}=2\left(R_{\text {out }}-R_{0}\right) U_{0} / V_{g}
\end{gathered}
$$

With constant flux $Q$. Reynolds number $R e_{g}$ is determined by hydraulic diameter, Digital images are acquired at 50 frames/seconds for 5 seconds. The stable shape of liquid bridge can be obtained by calculating the mean value of these images. Repeat above process for each gas flux. 


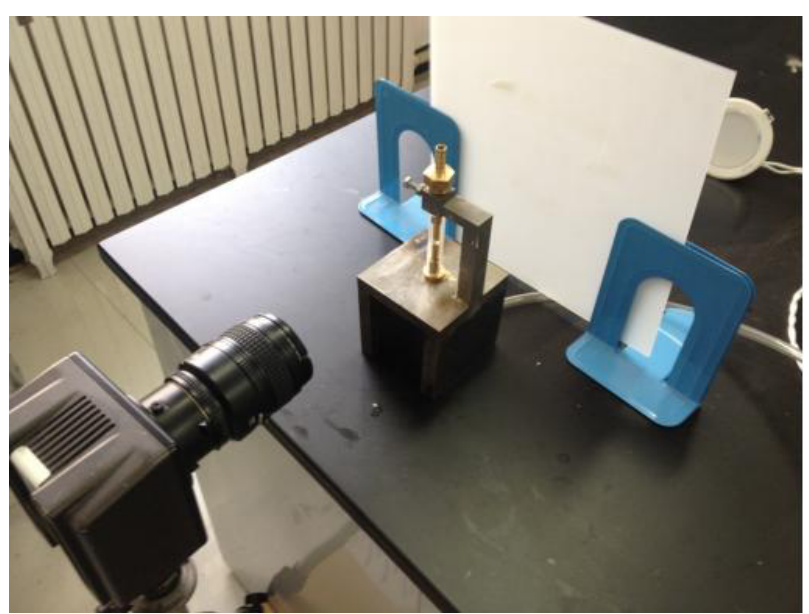

Figure 1. Actual picture of experimental set-up

\section{Results and Discussion}

The free surface contour is determined by the physical parameters of bilateral liquid and geometric parameters of the system. But generally, the effect of flow on the dynamic deformation of free surface is usually described by the capillary number $C a^{[5]}$.

$$
\begin{gathered}
\mathrm{Ca}=6 . \mathrm{O} \times 1 \mathrm{O}^{-4} U_{o} G^{2} \tilde{f}(V) \\
\mathrm{Ca}=v_{g} \rho_{g} U_{o} / \delta f\left(R_{0}, R_{o u t}, d, V\right)
\end{gathered}
$$

The coefficient is determined by the volume ratio $V$ and the gas velocity $U_{0}$. When the aspect ratio is $\Gamma=1$, the coefficient decreases from 1 to 0.5 with the volume ratio increasing from 0.8 to 1.07 . The capillary number increases with the airflow velocity increasing from $1 \mathrm{~m} / \mathrm{s}$ to $2 \mathrm{~m} / \mathrm{s}$. The predicted dynamic deformation of the free surface is $1.8 \mu \mathrm{m}-3.6 \mu \mathrm{m}$.

$$
\delta F_{c a}=\left(F-F_{0}\right)^{2} C a R_{0}
$$

This paper researches the dynamic deformation of free surface of the liquid bridge caused by the shear airflow. In the experiment, control parameters include the air flow, the ventilation direction, the height, and volume of the liquid bridge.

Figure 2 shows the dynamic deformation of free surface. For volume ratio $V=0.802$ and aspect ratio $\Gamma=1.0$, the upper part of liquid bridge shrinks and the lower part bulges. The reduction magnitudes of shrinkage and bulge are almost equal. The maximum and the minimum values are symmetric with respect to the middle which is near the height of $Z=1.5$. The shape of these curves is similar to the sine shape. The amplitude of the free surface deformation increases with the increase of gas velocity.

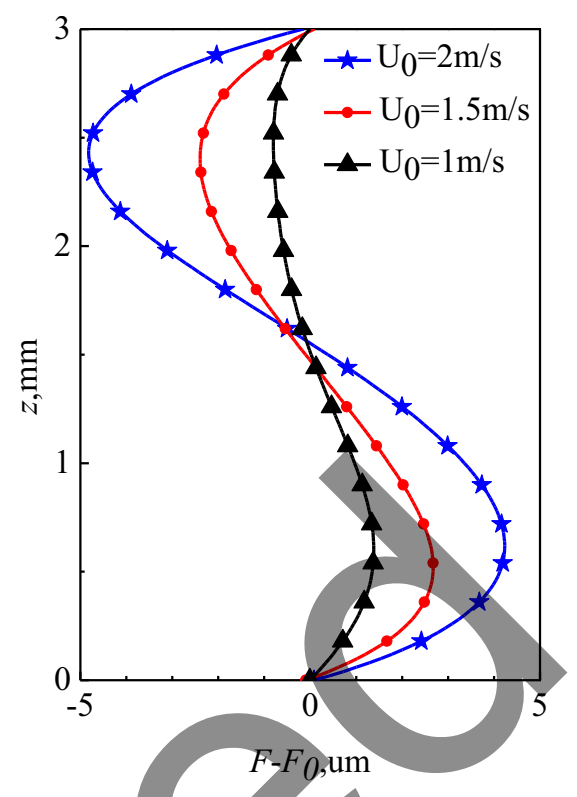

Figure 2. Dynamic interface deformation $\left(F-F_{0}\right)$ for different shear stresses for volume ratio $V_{1}=0.802, \Gamma=1.0$

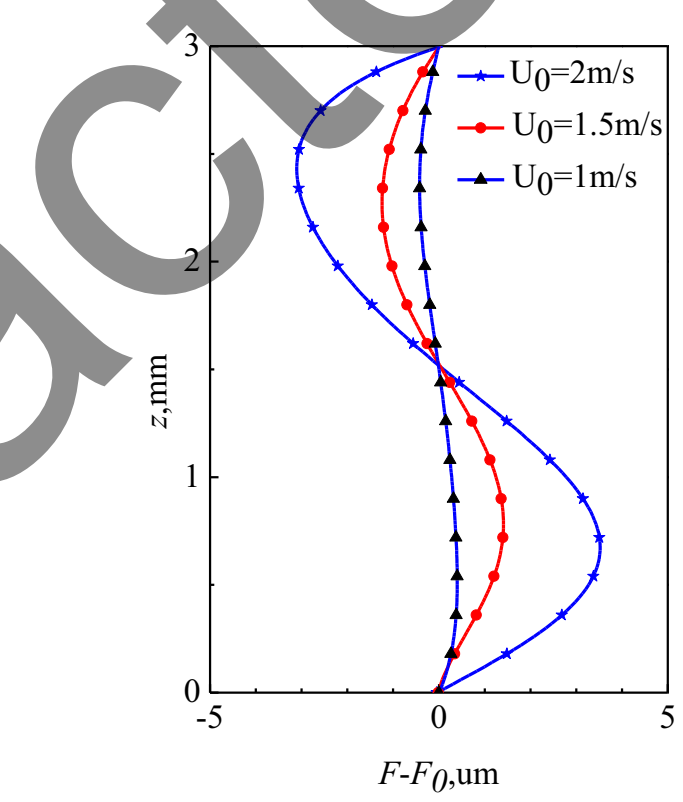

Figure 3. Dynamic interface deformation $\left(F-F_{0}\right)$ for different shear stresses for forvolume ratio $V_{1}=0.842, \Gamma=1.0$

Figure 3 shows the position difference of the free surface between the initial and current moment. The curves are similar to the volume ratio is $V=0.802$, but the amplitude maximum is comparatively smaller. Figure 4 illustrates this sinusoidal shape more obvious than Fig. 3.

Figure 5 shows that for large volume ratio $V=1.071$ $\left(F-F_{0}\right)$, the amplitude increases with the increase of the velocity. These curves present a double-peak pattern, which is different from the sine curve of the small volume ratio. For different velocities, the location of the first amplitude maximum along the flow direction is almost the same. 


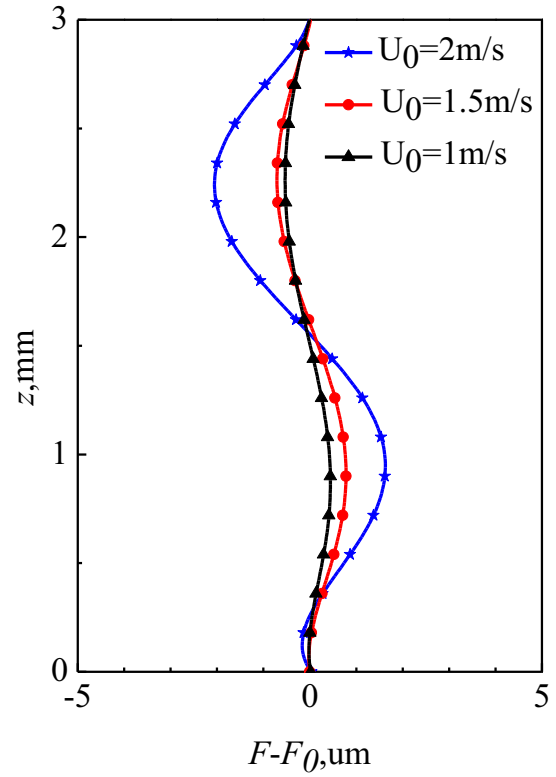

Figure 4. Dynamic interface deformation $\left(F-F_{0}\right)$ for different shear stresses for volume ratio $V_{1}=0.981, \Gamma=1$.
The results are summarized in Fig. 6, Fig. 7, and Fig 8. For three different velocities, the dynamic deformation of free surface $\left(F-F_{0}\right)$ is a function regarding to the volume ratio. From Fig. 6, the free surface is symmetric in the dynamic deformation only when the volume ratio is small. The greater the velocity is, the larger the maximum of the dynamic deformation is. The amplitude minimum of dynamic deformation occurs in the critical volume ratio of $V_{\mathrm{b}}$.

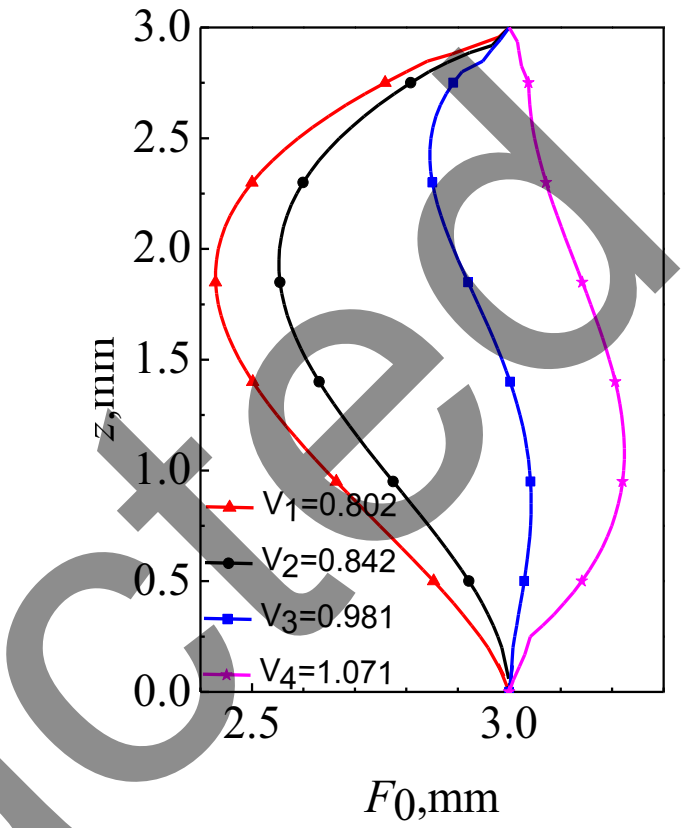

Figure 6. Position of free surface in different volume when liquid bridge is static

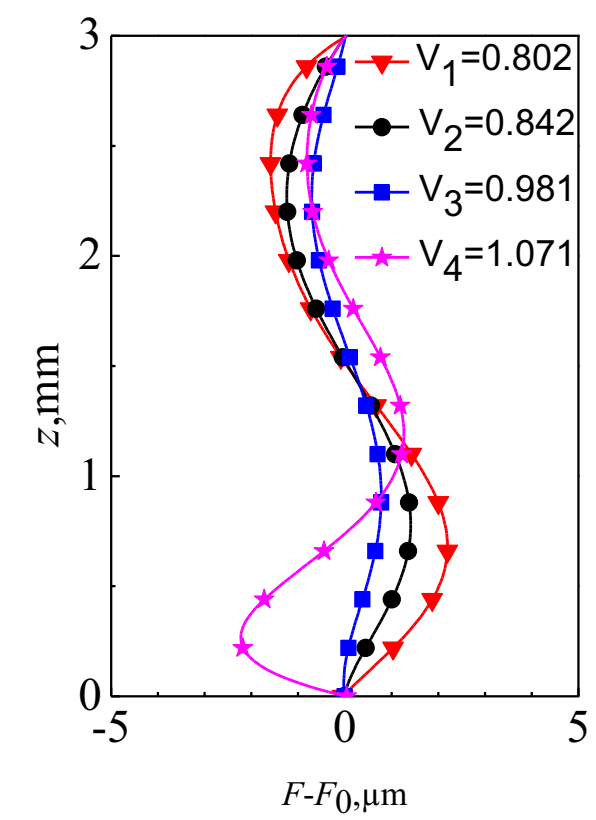

Figure 7. Dynamic interface deformation ratio $\left(F-F_{0}\right)$ when gas enters from the bottom $U_{0}=1.5 \mathrm{~m} / \mathrm{s}$ for $\Gamma=1.0$

From Fig. 8, it is found that when the gas enters from the bottom, the extreme values of the dynamic deformation at upper (circles) and lower (triangles) parts of a liquid bridge is a function of volume ratio $V$. The 
curves with different symbols (in order by the distance from the axis) correspond to the gas velocity $U_{0}=2 \mathrm{~m} / \mathrm{s}$, $1.5 \mathrm{~m} / \mathrm{s}$ and $1 \mathrm{~m} / \mathrm{s}$, respectively.

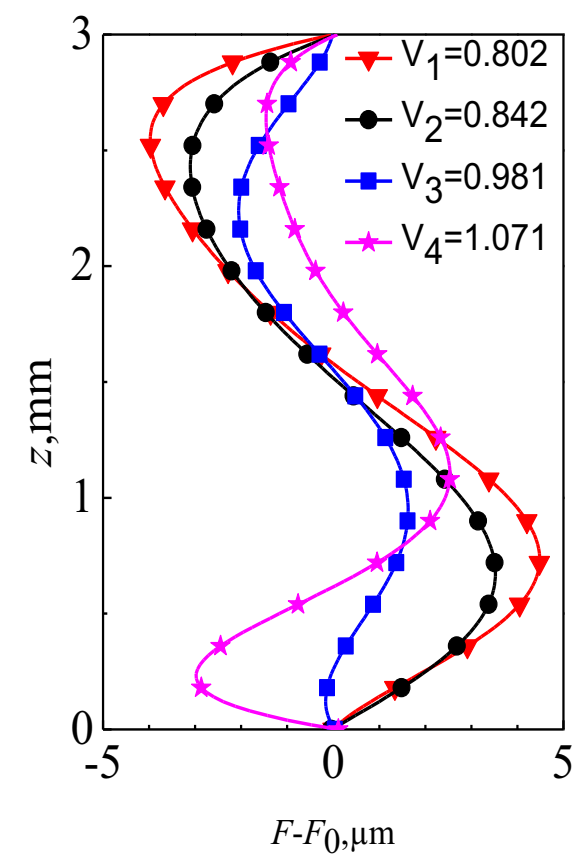

Figure. 8 Dynamic interface deformation $\left(F-F_{0}\right)$ when gas enters from the bottom $U_{0}=1.5 \mathrm{~m} / \mathrm{s}$ for $\Gamma=1.0$

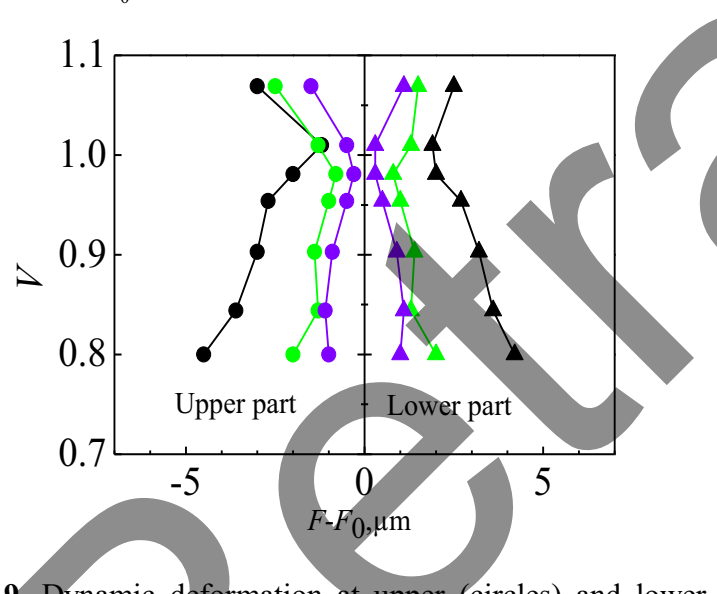

Figure 9. Dynamic deformation at upper (circles) and lower (triangles) parts of a liquid bridge for $\Gamma=1.0$

\section{Conclusions}

In this paper, the effect of the shear flow on the dynamic deformation of the free surface is studied. Liquid bridge is wrapped in a cylindrical glass sleeve. The dynamic deformation of the free surface of the liquid bridge is caused by the air flow through the annular glass sleeve. The following conclusions are obtained by experiment:

1. For small volume ratios, the airflow reduces the diameter of liquid bridge neck. Due to the mass conservation, the lower part bulges to balance the upper concave part. The difference curve of free surface position between current moment and initial moment is almost a sinusoidal curve. The maximum and the minimum values are symmetric with respect to the middle. The amplitude of surface deformation is linear with the velocity. The larger the volume ratio is, the smaller the amplitude is.

2. For large volume ratios, different places have different deformation amplitudes. The amplitude increases with larger velocity. The curves present a double-peak pattern, which is different from the sinusoidal curve of small volume ratios. For different velocities, the location of the first maximum amplitude along the direction of airflow is the same. The amplitude is linear with the tangible velocity, which is due to the mutual effect of gravity and airflow.

3. For large volume ratios, the airflow is hindered by the convex part of liquid bridge to resist the static force. Consequently, the fluid of the bottom moves to a certain position where the airflow effect can't resist the static force. This position is almost near the middle part where the liquid bridge bulges. Contrary to the law of small volume ratios, here, the larger the volume ratio is, the larger the amplitude is.

\section{Acknowledgements}

The present work is supported financially by the National Natural Science Foundation of China under the grants of 51376040 and 11072057

\section{References}

Yu. Gaponenko, I. Ryzhkov, and V. Shevtsova, On flows driven by mechanical stresses in a two-phase system. Fluid. Dyn. Mater. Process 6, 75 (2010)

2. Y. Gaponenk, A. Mialdun, and V. Shevtsova, Liquid entrainment by gas flow along the interface. Eur. Phys. J. E. 192, 63 (2011)

3. Y. Gaponenko, S. Glockner, A. Mialdun, and V. Shevtsova, Study of a liquid bridge subjected to interface shear stresses. Acta Astron.69 119(2011)

4. T. Matsunaga, A.Mialdu, K. Nishino, and V. Shevtsova, Measurements of gas/oil free surface deformation caused by parallel gas flow. Phys. Fluids 24, 062101 (2012)

5. S. Frank and D. Schwabe, Temporal and spatial elements of thermocapillary convection in a liquid zone. Exp. Fluids 23, 462 (1997)

6. H. C. Kuhlmann, Thermocapillary Convection in Models of Crystal Growth. Springer-Verlag. J E. (1999)

7. V. Shevtsova, Thermal convection in liquid bridges with curved free surfaces: Benchmark of numerical solutions. J. Cryst. Growth 280, 632 (2005)

8. M. Lappa, R. Savino, and R. Monti, Threedimensional numerical simulation of Marangoni instabilities in non-cylindricalliquid bridges in microgravity, Int. J. E. Heat Mass. Transfer 44,1983 (2001) 